\title{
Assessing the Influence of Landmarks and Paths on the Navigational Efficiency and the Cognitive Load of Indoor Maps
}

\author{
Hao Fang ${ }^{1}{ }^{1}$, Shiwei Xin ${ }^{1}$, Yanlin Zhang ${ }^{2}$, Zhong Wang ${ }^{1}$ and Jing Zhu ${ }^{3, *}$ \\ 1 Department of Digital Media, School of Art and Communication, China University of Geosciences, \\ Wuhan 430074, China; fanghao@cug.edu.cn (H.F.); xinshiwei@cug.edu.cn (S.X.); \\ wangzhong0820@cug.edu.cn (Z.W.) \\ 2 College of Mechanical and Electrical Engineering, Northeast Forestry University, Ha'erbin 150040, China; \\ yanlin@nefu.edu.cn \\ 3 Department of Network Engineering, School of Computer Science, Hubei Key Laboratory of Intelligent \\ Geo-Information Processing, Wuhan 430074, China \\ * Correspondence: zhujing@cug.edu.cn
}

Received: 27 November 2019; Accepted: 27 January 2020; Published: 31 January 2020

\begin{abstract}
The aim of this study was to investigate whether different expressions of landmarks and paths have different influences on the navigational efficiency and the cognitive load of indoor maps. The study tested 80 subjects by indoor path discovery experiments and measured their cognitive loads with the Cooper-Harper scale. According to the results, we extracted some key landmarks from all landmarks of the experimental indoor map and evaluated the saliency degree of each path. Then, the study tested subjects with four different types of experimental indoor maps by path-recognition tasks. The results showed that maps with key landmarks are more effective in terms of navigation than those with full landmarks, but there were no significant differences between their cognitive loads. Maps with highlighted paths are more effective in terms of navigation, and their cognitive loads are much lower than those without highlighted paths. In addition, this study found that women's cognitive loads were more affected by key landmarks compared with men's, while highlighted paths had no significant differences in terms of navigational efficiency and the cognitive load between males and females. Implications of these results with respect to the optimization strategies of indoor map designs are discussed.
\end{abstract}

Keywords: indoor map; key landmark; highlighted path; navigation efficiency; cognitive load; Cooper-Harper scale

\section{Introduction}

As the information carrier of location services, indoor maps are widely used to provide navigation to users [1]. In contrast to outdoor spaces, indoor spaces have some special characteristics such as smaller scales, more information volumes, complex space structures, enclosed spaces, clear segmentation and only human elements [2]. These characteristics determine different cognitive modes of map users for indoor and outdoor maps. The relative coordinates of "front, back, left and right" are used in indoor maps, and the absolute coordinates of "east, south, west and north" are used in outdoor maps [3]. Therefore, the traditional expression design of outdoor maps is not fully applicable to indoor maps. It is becoming an important task to explore how different elements of indoor maps impact map cognition, as well as to establish some new element expressions appropriate for indoor maps.

In existing research results, some indoor map design patterns have attracted a great deal of attention. Klipped [4] proposed the indoor emergency map design principle of "you-are-here-maps" 
by referring to the expression form of architectural drawings. However, this principle is not completely applicable to other indoor map forms. Arto Puikkonen [5] found that the minimalist design of indoor maps can effectively reduce users' cognitive loads. Nossum [6] designed a two-dimensional "pipeline map" and a three-dimensional simplified "pipeline map" of indoor maps by referring to the representation of metro maps. However, these designs have not yet been tested and evaluated. Based on spatial cognition theory and map perception theory, some scholars have put forward some indoor map design principles and element expression modes. But these design principles focused on the macroscale; they were difficult to test and evaluate $[2,3,7]$. These research works into indoor map design have made some explorations from different perspectives, but this research area is still at a preliminary stage in the field of design expression. Furthermore, lacking a uniform design theory and design principles have caused uneven qualities and unusable details of indoor maps. High cognitive loads and low navigational efficiencies are two serious problems in indoor map designs which need to be overcome $[7,8]$.

Landmarks and paths are two kinds of navigational clues for maps, and they both have important impacts on placating users' emotions to ensure correct decisions in path finding [9]. Landmarks show the spatial information of real objects, while paths simplify the navigation information which map users need to comprehend. However, the validity of both landmarks and paths is closely related to their sufficient information volumes and expression modes. Only the modes expressing prominently essential information selected from navigational processes can improve the spatial reasoning efficiency of indoor maps, reduce users' cognitive loads and promote users' navigation efficiency.

Taking the indoor map interface as the research subject, and based on the theory of spatial path identification, this study explores how design elements-mainly including key landmarks and highlighted paths-impact users' navigation efficiency and cognitive load. The study tested 80 subjects (40 males, 40 females) with indoor path-finding tasks. By analyzing the test results, we extracted some key landmarks from all landmarks and evaluated the saliency degree of every path. We collected the key landmarks and significant paths to determine experimental material maps. Then, the study tested the completion time and the cognitive load of users under controlled conditions through behavior experiments. We tested all subjects by means of path-recognition tasks to investigate whether landmarks (key landmarks, full landmarks) and paths (with highlighted, without highlighted) impact the navigational efficiency, the cognitive load and the gender interaction in indoor map designs. According to the analysis of the results, this paper deeply explores the principles of people's indoor path-finding behaviors, as well as revealing the cognitive mechanism of landmark and path expressions in indoor navigation. Furthermore, we provide optimization strategies for the design of indoor maps.

\section{Related Work}

\subsection{Landmark}

A landmark is a particular fixed object or area in a map; it plays an important role in path-finding processes. As a path guidance or a positional identification, it can help people to build positive representations of the surroundings, or improve their confidence in path-searching decisions. It is the most important clue in navigation $[9,10]$.

A landmark is defined by the following three attributes:

Visual attraction. Landmarks are visual attractions, mainly because of their sharp contrasts to surroundings and their prominent spatial positions. Researchers assess whether landmarks have visual attractions in terms of four aspects: the area, shape, color and visibility.

Semantic attraction. This attribute defines the meaning of a feature, mainly in terms of the cultural and historical significance contained in landmarks, and describes the clarity of landmarks.

Structural attraction. This defines the importance of the landmark's geometric features, such as intersections and boundaries [11]. 
However, importance of these three attributes are not the same. Different types of landmarks play different roles in navigation, and people tend to pay more attention to those which affect routing decisions. Redundant landmarks will interfere with users' decisions in path finding. Redundant information can assist users to make decisions in path findings, but it is not necessary. For example, "turn left at the next traffic light (necessary information), there is a bar around the corner (redundant information)." Compared to path instructions with information overload, instructions only with essential information can be read more effectively [12]. Therefore, this study hypothesizes that displaying landmarks selectively instead of displaying all landmarks can reduce the information load and the cognitive load of users. It can improve the reading efficiency of maps effectively.

This paper proposes two hypotheses:

Hypothesis 1a. The navigational efficiency of maps with key landmarks is significantly higher than that of maps with full landmarks.

Hypothesis 1b. The cognitive load of maps with key landmarks is significantly lower than that of maps with full landmarks.

\subsection{Path}

A path is a topological presentation of an indoor map. Paths can reduce the cognitive load of users, assist users to understand maps structurally and improve their navigation efficiency [13]. The figure-ground theory divides a visual sense (or a perceptual field) into two parts: many figures and a background. People tend to regard highlights as being perceived visually; i.e., the object they focus on, or a "Figure," when they observe things around them. In contrast, they regard parts of environments as "Background," with which they are less concerned. That means that "Background" is a counterpart to "Figure" [14]. This paper believes that highlighted key elements of indoor maps are better adapted to the spatial cognitive model of users, as well as improving the spatial reasoning efficiency and the expression rationality of spatial information. Paths are important expression elements of indoor maps. However, there is no indoor map product yet with a function of highlighting important paths. Researching and evaluating influences of highlighted indoor map paths on the navigational efficiency and the cognitive load of users can provide new strategies for indoor map design. Therefore, the following hypotheses are proposed in this paper:

Hypothesis 2a. The navigational efficiency of maps with highlighted paths is significantly higher than that of maps without highlighted paths.

Hypothesis $\mathbf{2 b}$. The cognitive load of maps with highlighted paths is significantly lower than that of maps without highlighted paths.

\subsection{Paths and Landmarks}

Both paths and landmarks are important expressive elements of indoor maps. They play different roles in navigation and complement each other. Considered from expression contents, a path is a topological representation of the spatial relationship in an indoor map, and it constructs the outline relationship for indoor factors in a graphical way, which can help users to understand the overall indoor environment. A landmark plays an important role in locating and explaining indoor map factors. It explains the content of indoor map factors in the form of signs and expresses the indoor map factors with spatial orientation. In terms of the expression form, landmarks are attached to paths, appearing with the emergence of paths. A map without landmarks would not express valid information, and a map without paths would not work as a map. A map can effectively express information only by integrating path and landmark information reasonably. Thus, the following research hypothesis is proposed: 
Hypothesis 3. Information volumes of landmarks and paths have interactive effects on the navigational efficiency and the cognitive load of users.

\subsection{Measuring the Navigational Efficiency and the Cognitive Load}

The navigational efficiency commonly refers to the time that users need to complete a positioning task by using maps. In this paper, the experimental tasks were to recognize paths that participants had passed through. The navigational efficiency was measured by recording the path-recognition time of users [15]. The cognitive load refers to the total amount of cognitive resources required for processing information in cognitive activities. Excessive cognitive loads will reduce the task completion performance of users [16]. The cognitive load, which needs to be controlled in the study, is affected by previous knowledge and experiences, genders, ages, spatial cognitive abilities and other factors [17]. Common subjective tools used to measure mental workloads include the Paas's nine-point mental effort rating scale (PAAS scale), Subjective Workload Assessment Technique (SWAT scale), NASA-Task Load Index (NASA-TLX scale) and modified Cooper-Harper scale. The theoretical basis of these subjective assessment methods is that the occupation of the learner's psychological resources is related to the individual's effort and the difficulty of the task, and the effort and difficulty of the task can be accurately expressed by the learner [18].

The PAAS scale uses a nine-point scoring system to assess cognitive load from two dimensions: psychological effort and task difficulty. Scores from one to nine mean that the psychological effort and the difficulty of the materials are increasing in order: one is the least effort and is very easy, five is medium effort and medium difficulty, and nine is very hard and very difficult. The total mental-load value is the mean of the two dimensions.

The SWAT scale consists of three dimensions, namely the time load, mental-effort load, and psychological stress. The time load reflects how much free time people have available during the task, the effort load reflects how much effort people need to perform the task, as well as the mental stress measures the psychological state manifestation of anxiety and dissatisfaction during the task degree. Each dimension is divided into three levels: light, medium and heavy, with a total of 27 combinations of the three dimensions.

The use of the SWAT scale for mental-load assessment is divided into two steps. The first phase is the scale-development phase, in which the researchers made 27 cards based on 27 combinations. Before evaluating the mental load, the subjects ranked the mental load represented by the 27 cards from small to large according to their feelings, and assigned them a score (1 to 27 points). They determined the importance of the three dimensions to their total mental load. Based on the relative importance of each dimension, six theoretical groups named by scrambled letters $\mathrm{T}$ (time load)-S (psychological stress load)-E (effort load) are formed, namely TES, TSE, ETS, EST, STE, and SET. Among them, TES shows that the time load is considered the most important by the subject, and the effort load is the second most important, while the stress load is the least important. During the evaluation, the corresponding group of subjects was determined according to the magnitude of the correlation coefficient between the order of the subjects and the six theoretical orders. The second stage is the scoring stage, in which the subjects select the level that is consistent with their actual situation in three dimensions according to the situation of completing the task. Based on the results of the selection of the subjects, the researchers combine the grouping at the development stage of the scale to find out the scores of the subjects' mental load and convert them to a point value from 0 to 100; the higher the score, the greater the mental load.

The NASA-TLX scale is also a multidimensional psychological-load assessment scale. The scale consists of six dimensions: mental demand (MD), physical demand (PD), time demand (TD), effort (EF), performance (PE) and frustration level (FR). Each dimension is represented by a straight line divided into 20 equal parts, and the straight lines are marked with low and high. Except for the performance level, the load of the remaining five dimensions gradually increases from left to right. The performance level from left to right is good to poor; that is, the lower the score, the better the 
performance level, and the lower the load. The calculation for the total psychological load (overall workload, OW) is as follows: a pairwise comparison method is used to pair six items to form 15 pairs. The participants are required to select each pair with the total psychological load. For the item that is most closely related, the weight of the item on the total psychological load is determined according to the number of times each item is selected. The total load is the weighted average of six entries.

The Cooper-Harper scale was the earliest tool that was applied to measure the users' cognitive load. The modified Cooper-Harper scale is an improved version of the Cooper-Harper scale. Due to the applicability of the scale in human-computer interface cognitive-load measurement and the ease of its use [19], this study measured the cognitive load of subjects by using the Cooper-Harper scale. The Cooper-Harper scale divides the difficulty of tasks into 10 grades, with the scale rating from 1 to 10. The scale adopts decision trees to evaluate the difficulty. A complete evaluation process has four steps [20]:

1. "Although mistakes may be serious and occur often, can the specified task be still completed in most cases?" If subjects answer "yes," the procedure enters step 2; if subjects answer "no," the task's difficulty grade is rated as 10 .

2. "There are few mistakes and it doesn't matter." If subjects answer "yes," the assessed difficulty grade is rated between 4 and 6, and the procedure enters step 3; if subjects answer "no," the assessed difficulty grade is rated between 7 and 9 , and the procedure enters step 4 directly.

3. "Acceptable levels of mental workload." If subjects answer "yes," the difficulty grade is rated between 4 and 6, and the procedure enters step 4; if subjects answer "no," the assessed difficulty grade is rated between 1 and 3 , and the procedure enters step 4 .

4. Each case is further subdivided to determine the final evaluation grade.

\section{Methods and Experiments}

\subsection{Subjects}

The 80 subjects in this study were selected by the MCT (Mental Cutting Test). MCT is an internationally recognized test of spatial cognitive ability and can fully investigate subjects' spatial cognitive abilities, including psychological rotation, psychological cutting, etc., by asking the subjects to describe the shape, edge length and angle of cut sections (Figure 1) [21].

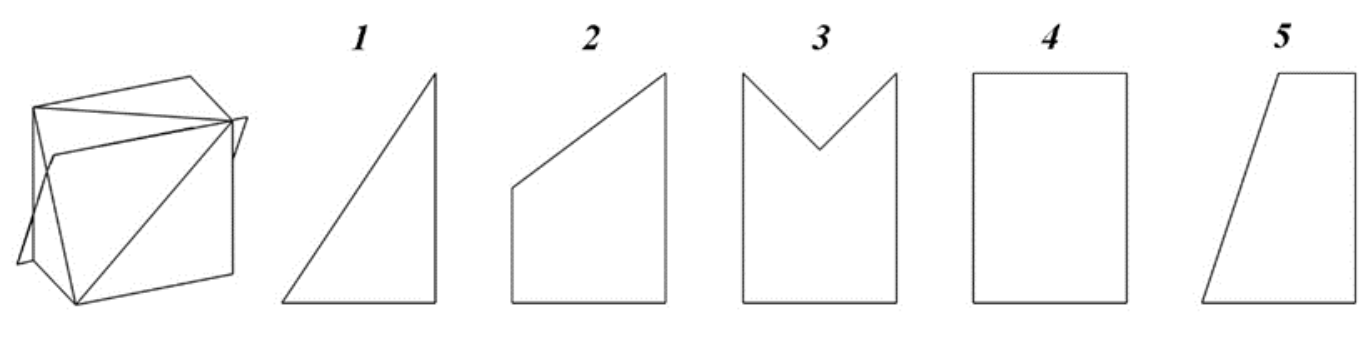

Figure 1. Mental Cutting Test.

A total of 80 subjects were divided into four groups on average. Each group had 10 males and 10 females. Each subject had the same spatial-cognitive-ability grade, and their ages were between 18 and 20. Each subject was completely unfamiliar with the experimental environment. All subjects were volunteers with normal eyesight or normal eyesight after correction. All subjects received a reward after the experiments.

\subsection{Experimental Design}

This study adopted the between-group design of 2 (landmarks: key landmarks and full landmarks) $\times 2$ (paths: highlighted and not highlighted). Subjects belonging to each group only completed one 
test of four. In all tests, landmarks and paths were regarded as independent variables, while the navigational efficiency and the cognitive load were regarded as dependent variables.

\subsection{Experimental Materials}

The study obtained landmark and path information of the test environment through behavioral experiments. It determined the key landmarks and highlighted path information according to the experiments. Four types of indoor maps were created by the study: full-landmark maps without highlighted paths, full-landmark maps with highlighted paths, key-landmark maps without highlighted paths and key-landmark maps with highlighted paths. The specific process was as follows.

\subsubsection{Extracting Key Landmarks}

The existing methods of landmark extracting experiments enable subjects to learn the predetermined experimental routes in the environment. They then require the subjects, who were asked to provide path descriptions or path sketches containing landmarks, to complete path-recognition tasks to assess the degree of importance of landmarks in path finding [22,23]. This experimental paradigm was adopted in the study to extract all landmarks for experimental materials. The Wuhan Optical Valley Plaza, a commercial pedestrian indoor street in the Hubei province of China, was chosen as the experimental environment of this study. After guiding subjects from one site to another site through the Wuhan Optical Valley Plaza, subjects were asked to recall the iconic objects, as much as they were able, which they had passed. Then, they ranked all the reference objects by quantities that met the requirements of the test. After that, the study selected key landmarks at a high rank that all subjects had recalled before. There were 54 kinds of 654 reference objects that were totally recalled by the participants, 52 kinds of 601 reference objects were maintained after eliminating invalid information. The top $80 \%$ of quantities of reference objects in rankings were selected as the key information. Therefore, 17 key landmarks were ultimately determined as a part of the experimental materials.

\subsubsection{Highlighted Paths Design}

The levels of path significance in outdoor maps are generally defined according to road grades, traffic flows or expected driving speeds [24]. This study regarded the flow of pedestrians as the indoor path salience, and referenced to the criteria in outdoor maps to divide grades [25].

Highlighted path designs screened the main indoor paths of the Optical Valley Plaza through behavioral experiments. The steps were as follows.

After identifying all intersections that subjects could pass by, this study set up monitoring points in the middle of every two contiguous intersections, with a total of 25 monitoring points (Figure 2a). Then, the researchers counted the flow of people every half an hour at each monitoring point, identifying the main paths with higher pedestrian flows based on the collected data, and finally formed a map with highlighted paths of the indoor streets (Figure 2b).

\subsubsection{Materials Design}

Taking landmarks (key landmarks or full landmarks) and paths (highlighted or not highlighted) as variables, four prototypes of indoor maps were created and colored by the ArcGIS Online (Geographic Information System Software) (Figure 3). 


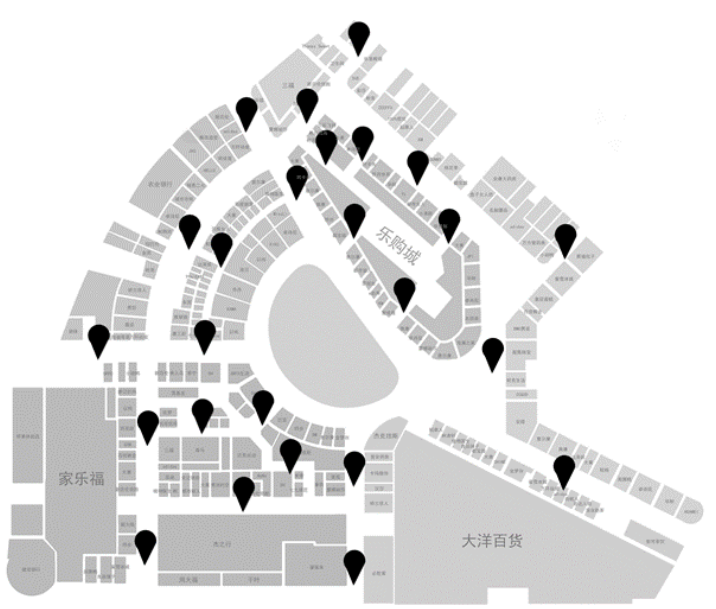

(a)

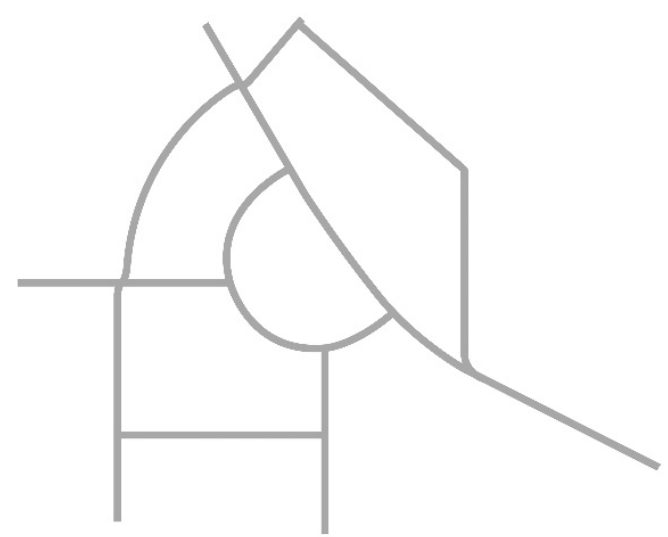

(b)

Figure 2. (a) The distribution of pedestrian flow test points and (b) highlighted paths of the experimental map.

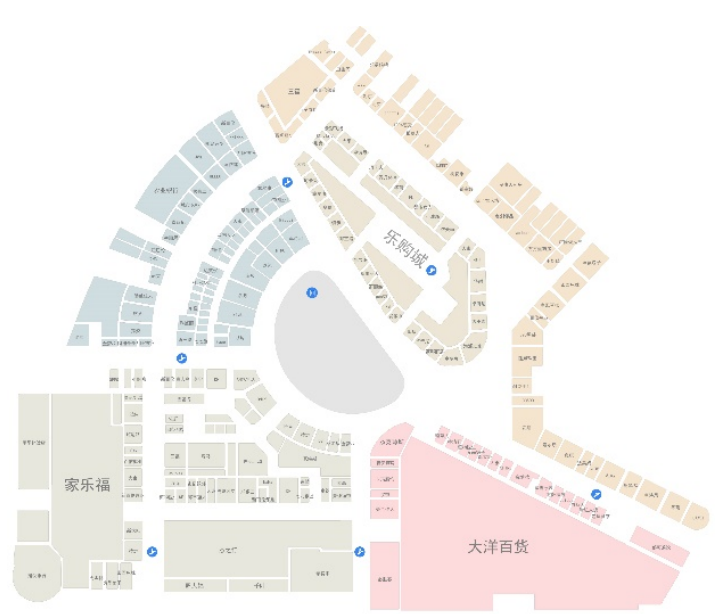

(a)

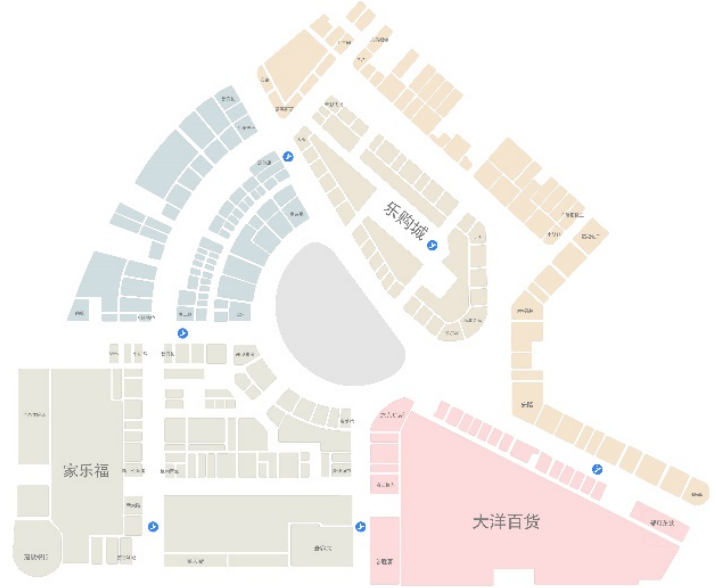

(c)

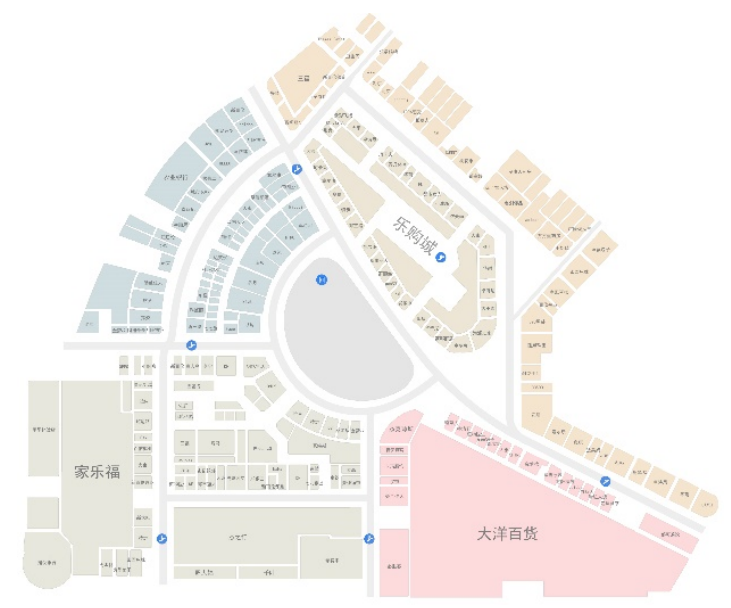

(b)

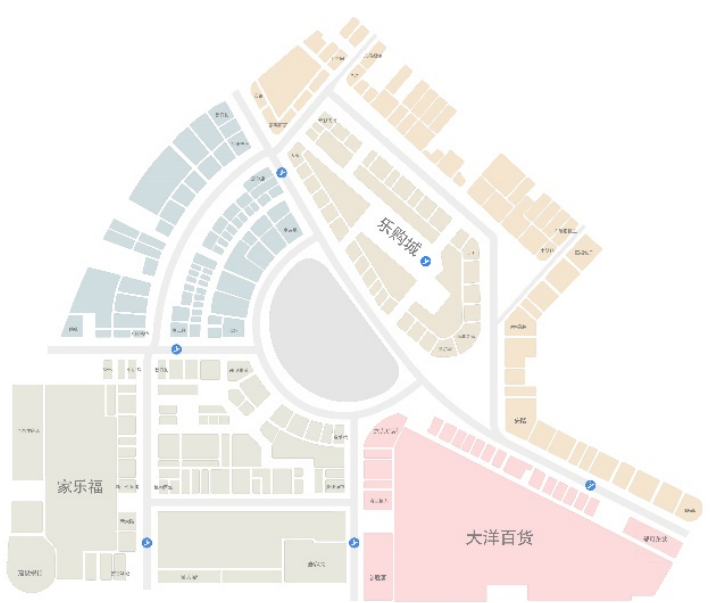

(d)

Figure 3. (a) The full-landmark map without highlighted paths, (b) the full-landmark map with highlighted paths, (c) the key-landmark map without highlighted paths and (d) the key-landmark map with highlighted paths. 


\subsection{Experiments}

\subsubsection{Experimental Devices}

The study used LED screens (resolution: $3840 \times 2160$ ) to display four kinds of indoor maps, used intelligent mobile phones to take photos to record the test processes, used electronic watches to record the time each subject spent on each task, and used the Cooper-Harper table to score the cognitive loads of subjects. The experiments were conducted at the China University of Geosciences.

\subsubsection{Navigation Efficiency Measurement}

According to the order of the previous grouping, subjects were invited to participate in the path-recognition tests of four kinds of indoor maps in four batches. After matching their names on a checklist, subjects were required to turn their backs to the screen. Then, the corresponding experimental materials were presented and played on the screen by researchers before subjects were asked to turn around. The task of the subjects was to search as quickly as possible for the route he (or she) had passed through as reflected on the map, pointing at it with fingers from the starting spot to the final spot. When a subject finished the task correctly, the total time he (or she) required would be recorded on a sheet. The collected data were used as the indication of the navigational efficiency of map users. Furthermore, the only information researchers could tell subjects was whether the subjects' results of the task were correct or not, but they could not give any hints or interruptions during the experiment processes.

\subsubsection{Spatial Cognitive Load Measurement}

Researchers assessed the cognitive loads of all subjects by using the Cooper-Harper scale. Subjects selected the description items which best conformed to their performance during the task. Moreover, researchers used the Cooper-Harper scale to quantify the subjects' spatial cognitive loads into different scores, which represented the task-finishing cognitive load in a statistical form.

After completing all tests, gifts as rewards were given to the subjects, and researchers bound the task-time-record sheets together with the relevant score sheets recording the spatial cognitive load and matched them to each name on the list of subjects.

\section{Results}

After collecting all test data, researchers removed invalid samples for those unfinished tasks to improve the credibility of the results. There were 19 valid samples in the group of the full-landmark map without highlighted paths, 20 valid samples in the group of the full-landmark map with highlighted paths, 18 valid samples in the group of the key-landmark map without highlighted paths and 17 valid samples in the group of the key-landmark map with highlighted paths. The total number of valid samples was 74 .

Statistical Product and Service Solutions (SPSS) Version 20.0 (Modified date: 17 June 2018) is statistical software that helps users in dealing with data. This study used the SPSS Version 20.0 to analyze all the valid data.

\subsection{Evaluation of the Navigational Efficiency}

The assessment of navigational efficiency can be indicated by navigational task-finishing time analysis and investigating whether there are intergroup differences caused by the various design expressions of indoor maps. The Independent Sample $t$ Test method was used for intergroup comparisons, and the results are as follows.

Table 1 shows that the task-finishing time of the group using the full-landmark map is significantly different from that of the group using the key-landmark map $(t=7.393, p=0.000)$, and the task time of the group using the full-landmark map $(\mathrm{M}=115.846, \mathrm{SD}=31.025)$ is significantly higher than that of 
the group using the key-landmark map $(\mathrm{M}=68.829, \mathrm{SD}=22.448)$. The task time of the group using the map with highlighted paths is significantly different from that of the group using the map without highlighted paths $(t=-3.302, p=0.000)$, and the task-finishing time of the group using the map with highlighted paths $(\mathrm{M}=80.649, \mathrm{SD}=32.392)$ is much smaller than that of the group using the map without highlighted paths $(\mathrm{M}=106.568, \mathrm{SD}=35.074)$.

Table 1. Independent Sample $t$ Test of task time.

\begin{tabular}{ccccccc}
\hline Independent Variable & Level & $\mathbf{M}$ & SD & $\boldsymbol{F}$ & $\boldsymbol{t}$ & $\boldsymbol{p}$ \\
\hline \multirow{2}{*}{ Landmarks } & Full Landmark & 115.846 & 31.025 & \multirow{2}{*}{3.028} & \multirow{2}{*}{7.933} & 0.000 \\
& Key Landmark & 68.829 & 22.448 & & & \\
Highlighted Paths & With & 80.649 & 32.392 & 0.416 & -3.302 & 0.001 \\
& Without & 106.568 & 35.074 & & & \\
\hline
\end{tabular}

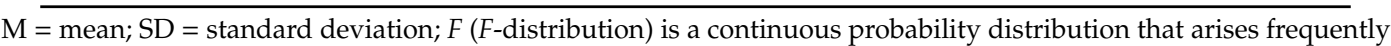
as the null distribution of a test statistic; $t$ is an inferential statistic; $p$ is the probability of obtaining test results as extreme as the results actually observed during the test; $\Delta \mathrm{M}$ is the changing value of means between two groups.

\subsection{Evaluation of the Spatial Cognitive Load}

The spatial cognitive-load analysis is performed to analyze the cognitive scores of all subjects collected during tests and determine if there are intergroup differences caused by different expressions of indoor maps. The Independent Sample $t$ Test method was also used for inter-group comparisons in the study, and the results are shown in Table 2.

Table 2. Independent Sample $t$ Test of cognitive load.

\begin{tabular}{ccccccc}
\hline Independent Variable & Level & M & SD & $\boldsymbol{F}$ & $\boldsymbol{t}$ & $\boldsymbol{p}$ \\
\hline \multirow{2}{*}{ Landmarks } & Full Landmark & 3.744 & 1.517 & \multirow{2}{*}{0.340} & 0.972 & 0.334 \\
& Key Landmark & 3.400 & 1.519 & & & \\
Paths & Highlighted & 2.784 & 1.294 & 0.003 & -5.291 & 0.000 \\
& Not highlighted & 4.378 & 1.299 & & & \\
\hline
\end{tabular}

Table 2 shows that there are no significant differences of cognitive loads between the group using the full-landmark map and the group using the key-landmark map $(t=0.972, p=0.334)$. The cognitive load of the group using the full-landmark map $(\mathrm{M}=3.744, \mathrm{SD}=1.517)$ is slightly larger than that of the group using the key-landmark map $(\mathrm{M}=3.400, \mathrm{SD}=1.519)$.

On the other hand, the cognitive load of the group using the map with highlighted paths is significantly different from that of the group using the map without highlighted paths $(t=-5.291$, $p=0.000)$, and the cognitive load of the group using the map with highlighted paths $(\mathrm{M}=2.784$, $\mathrm{SD}=1.294)$ is significantly lower than that of the group using the map without highlighted paths $(\mathrm{M}=4.378, \mathrm{SD}=1.299)$.

\subsection{Interaction Effects between Landmark Display Mode and Highlighted Paths}

The results of the Independent Sample $t$ Test have shown that both landmark and path expressions have certain impacts on subjects' navigational efficiency and cognitive load. On this basis, this study further investigated whether there are interaction effects between expressions of landmarks and paths on the navigational efficiency and the cognitive load of users through the method of two-dimensional intergroup variance analysis. The results are shown in Tables 3 and 4.

Table 3. Results of cognitive-load interaction test.

\begin{tabular}{ccccc}
\hline Source & Dependent Variable & Mean Square & $\boldsymbol{F}$ & $\boldsymbol{p}$ \\
\hline $\begin{array}{c}\text { Paths (highlighted, not highlighted) } \times \\
\text { Landmarks (key landmarks, full landmarks) }\end{array}$ & Cognitive Load & 0.012 & 0.007 & 0.934 \\
\hline
\end{tabular}


Table 4. Results of task-time interaction test.

\begin{tabular}{ccccc}
\hline Source & Dependent Variable & Mean Square & $\boldsymbol{F}$ & $\boldsymbol{p}$ \\
\hline $\begin{array}{c}\text { Paths (highlighted, not highlighted) } \times \\
\text { Landmarks (key landmarks, full landmarks) }\end{array}$ & Task Time & 828.921 & 1.480 & 0.228 \\
\hline
\end{tabular}

Tables 3 and 4 show that paths and landmarks have no significant interactions with the cognitive load $(F=0.007, p=0.934)$, and there are no significant interactions on the task time between paths and landmarks $(F=1.480, p=0.228)$.

\subsection{Impacts of Landmark and Path Expressions on Gender}

The mean change of the cognitive load and the task time of indoor map users reflects the degree of influences that landmark and path expressions have on gender.

Table 5 shows that the mean change of landmarks for females $(\Delta \mathrm{M}=0.570)$ is greater than that of males in terms of the cognitive load $(\Delta \mathrm{M}=0.128)$. This result indicates that the change in the landmark expression affects females more than males in the cognitive load. The table also shows the mean change of highlighted paths for males $(\Delta \mathrm{M}=1.947)$ is significantly greater than that of females $(\Delta \mathrm{M}=$ 1.222). This result means that the highlighted path has a greater impact on males than females in the cognitive load, in terms of the task-finishing time. Furthermore, the landmark mean change of females $(\Delta \mathrm{M}=48.081)$ is scarcely different from that of males $(\Delta \mathrm{M}=45.961)$, which indicates the effects of the landmark expression on the navigational efficiency is almost the same for males and females. Finally, the highlighted path mean change for males $(\Delta M=31.947)$ is significantly larger than that of females $(\Delta \mathrm{M}=19.556)$, which means that the impact of the highlighted path on the navigational efficiency of males is greater than that of females.

Table 5. Changes in cognitive score mean and task time mean.

\begin{tabular}{cccccccc}
\hline $\begin{array}{c}\text { Dependent } \\
\text { Variable }\end{array}$ & Gender & $\begin{array}{c}\text { Full } \\
\text { Landmark }\end{array}$ & $\begin{array}{c}\text { Key } \\
\text { Landmark }\end{array}$ & $\mathbf{\Delta M}$ & $\begin{array}{c}\text { Without } \\
\text { Highlighted } \\
\text { Paths }\end{array}$ & $\begin{array}{c}\text { With } \\
\text { Highlighted } \\
\text { Paths }\end{array}$ & $\mathbf{\Delta M}$ \\
\hline Cognitive & Male & 3.350 & 3.222 & 0.128 & 4.263 & 2.316 & 1.947 \\
Load & Female & 4.158 & 3.588 & 0.570 & 4.500 & 3.278 & 1.222 \\
Task Time & Male & 106.850 & 60.889 & 45.961 & 101.053 & 69.105 & 31.947 \\
& Female & 125.316 & 77.235 & 48.081 & 112.390 & 92.833 & 19.556 \\
\hline
\end{tabular}

\section{Discussion}

The results show that the expressions of landmarks (key landmarks, full landmarks) and paths (highlighted, not highlighted) have different effects on the navigational efficiency and the cognitive load of indoor map users, but the ways that they impact the navigational efficiency and the cognitive load are not exactly the same.

On the premise that the gender, age, spatial familiarity, spatial cognitive ability and environment are same, there are no differences between full-landmark maps and key-landmark maps in reducing users' cognitive load. According to the visual selective attention theory, visual information processing is selective; the targets with effective cue prompts would be paid attention to first, and users will generally ignore the interference information which is irrelevant to tasks [26]. Related study results have shown that establishing hierarchical visual knowledge presentation is helpful to reduce the disorder visual sense in man-machine interfaces as well as reduce the volume of load input in the cognitive process [27]. In path-recognition tasks, participants paid more attention to those landmarks including beginning spots, attractive spots or corner points along paths. As a result, there were no significant differences between full-landmark maps and key-landmark maps on cognitive loads. The highlighted path has a significant effect on reducing the spatial cognitive load of people in indoor navigation. Compared with 
maps without highlighted paths, maps with highlighted paths can reduce the cognitive load of users by $27 \%$. In this study, compared to the map without highlighted paths, the map with highlighted paths can reflect the topological graphic structure of the indoor space more intuitively and clearly, enhancing the users' hierarchical sense to help them with a structured comprehension of the indoor space as well as reducing the cognitive load.

Under the premise that the gender, age, spatial familiarity, spatial cognition and environment remained the same, maps with key landmarks will improve people's indoor space navigation efficiency nearly $40 \%$ more than a full-landmark map. In path-recognition experiments, the subjects were asked to point out the integrated path from the origin to the destination by their fingers, and it took more time when subjects used full-landmark maps. It could be assumed that the main reason was that full-landmark maps had more interferential landmark information around the path, which delayed the task-finishing time. Highlighted-path maps will also improve people's indoor space navigation efficiency. Compared with maps without highlighted paths, maps with highlighted paths can improve the navigational efficiency of users by nearly $25 \%$. As a cognitive activity, the efficiency of map reading would be affected by the cognitive load. Thus, compared to maps without highlighted paths, maps with highlighted paths can effectively reduce the cognitive load of people.

Some studies have suggested that the effects of landmarks and paths on subjects' attention are independent of each other at different stages when taking a cognitive task, and there are no significant interactions [28]. There are also no interactions between landmarks and paths in affecting users' navigation efficiency and cognitive load, which means that the landmark expressions have no influence on the navigational efficiency, or the cognitive load does not experience an effect from the highlighted paths. In this study, by paying subjects for return visits, it is found that they generally looked for the starting of the path at first, and then made a decision according to the information around the path in the process of the indoor location; that is, subjects have different attention preferences at different stages of a task. The Spotlight Model argued that the human brain cannot effectively process all information of stimuli within the range of vision simultaneously. This theory regarded the concentration as a spotlight, which is the only area which can be seen clearly. When stimuli fall outside the spotlight area, their information could be ignored and hardly analyzed, while those stimuli inside the spotlight area could be processed effectively and perceptually.

The results of this study show that different landmark expressions have greater effects on females' cognitive load than that of males. There are no significant differences between males and females in terms of navigational efficiency. Highlighted paths have a greater impact on both the cognitive load and the navigational efficiency of men than women. The results are consistent with the research results of Yan et al. [29]. In the process of transforming graphic maps into mental maps, males are more sensitive to spatial relationships, while females are more sensitive to detailed information such as signs and locations; thus, landmark expressions have greater impacts on females, and highlighted paths have greater impact on males.

\section{Conclusions}

Through path-recognition tasks, this study investigated how landmarks (key landmarks, full landmarks) and paths (highlighted, not highlighted) affect users' navigational efficiency and cognitive load in indoor map design, and investigated the underlying reasons for these effects. Four conclusions are drawn as follows: 1) the navigational efficiency of indoor map users using key-landmark maps is obviously higher than that of users using full-landmark maps, and the navigational efficiency of indoor map users using maps with highlighted paths is obviously higher than that of users using maps without highlighted paths; 2) there are no significant differences between full-landmark maps and key-landmark maps in cognitive loads, and the cognitive load of maps with highlighted paths is much lower than that of maps without highlighted paths; 3 ) there are no interactions between landmark and path expressions in terms of the navigational efficiency or the cognitive load; 4) the expression of landmarks has a greater impact on the cognitive load of females than males, but there are 
no differences between females and males in terms of navigational efficiency, while highlighted paths have a greater impact both on the cognitive load and the navigational efficiency of males than females.

This study assessed the influence of indoor map elements on the navigational efficiency and the cognitive load by using a quantitative method, broadening the research perspective and research methods of indoor map design, providing new theoretical support for indoor map design and optimizing the representation of indoor maps. The results of this study would have some significance for improving the expression efficiency of indoor map geographic information.

However, there are some limitations of this study. Limited by the project resources and the experiment requirements, the study selected subjects only from freshmen of the university, who almost have a single identity and age. Furthermore, the study only used an indoor commercial space as the test environment and did not vary the indoor space types to research indoor map design in more depth. Users may have diverse cognitive modes and varied mental pressure levels in the different types of indoor places. In the future, we will study and verify whether the conclusions of this study could be adapted to other ages, identities and indoor sites.

Author Contributions: Conceptualization, Hao Fang and Jing Zhu; Methodology, Hao Fang; Software, Zhong Wang; Validation, Shiwei Xin; Formal Analysis, Shiwei Xin; Investigation, Shiwei Xin; Resources, Zhong Wang and Shiwei Xin; Data Curation, Yanlin Zhang; Writing-Original Draft Preparation, Hao Fang, Jing Zhu and Zhong Wang; Writing — Review and Editing, Jing Zhu and Shiwei Xin; Visualization, Zhong Wang; Supervision, Hao Fang; Project Administration, Jing Zhu; Funding Acquisition, Jing Zhu. All authors have read and agreed to the published version of the manuscript.

Funding: This research was funded by [Open Research Project of The Hubei Key Laboratory of Intelligent Geo-Information Processing] grant number [KLIGIP-2017B11], [China University of Geosciences Research Project on Teaching Reform of Postgraduate Education] grant number [YJG2018217] and [Special Funds for Fundamental Research Business Expenses of Central Universities] grant number [2572019BF07]. And the APC was funded by [Open Research Project of The Hubei Key Laboratory of Intelligent Geo-Information Processing] grant number [KLIGIP-2017B11].

Conflicts of Interest: The authors declare no conflict of interest.

\section{References}

1. Al Hammadi, O.; Al Hebsi, A.; Zemerly, M.J.; Ng, J.W. Indoor localization and guidance using portable smartphones. In Proceedings of the 2012 IEEE/WIC/ACM International Conferences on Web Intelligence and Intelligent Agent Technology, Macau, China, 4-7 December 2012.

2. Zhou, Z.; He, Z.; Miao, J. Design and Representation of Indoor Map. Bull. Surv. Mapp. 2016, 10, 39-44.

3. Tian, V.; Zhou, C.; Xi, C. Study and Practice of Indoor Map representation Method. J. Surv. Mapp. Sci. Technol. 2014, 31, 635-640.

4. Klippel, A.; Freksa, C.; Winter, S. You-are-here maps in Emergencies -the Danger of Getting lost. J. Spat. Sci. 2006, 51, 117-131. [CrossRef]

5. Puikkonen, A.; Sarjanoja, A.H.; Haveri, M.; Huhtala, J.; Häkkilä, J. Towards Designing Better Maps for Indoor Navigation Experiences from a Case Study. In Proceedings of the 8th International Conference on Mobile and Ubiquitous Multimedia, Cambridge, UK, 22-25 November 2009.

6. Nossum, A.S. IndoorTubes A Novel Design for Indoor Maps. Am. Cartogr. 2011, 38, 192-200. [CrossRef]

7. Deng, C.; Tian, J.; Xia, Q. New model of interior map design and expression for mobile terminals. J. Syst. Simul. 2017, 12, 2952-2963.

8. Lorenz, A.; Thierbach, C.; Baur, N.; Kolbe, T.H. Map Design Aspects, Route Complexity, or Social Background? Factors Influencing user satisfaction with indoor navigation maps. Cartogr. Geogr. Inf. Sci. 2013, 40, 201-209. [CrossRef]

9. May, A.J.; Ross, T.; Bayer, S.H.; Tarkiainen, M.J. Pedestrian Navigation Aids: Information Requirements and Design Implications. Pers. Ubiquitous Comput. 2003, 7, 331-338. [CrossRef]

10. Millonig, A.; Schechtner, K. Developing Landmark-Based Pedestrian-Navigation Systems. Intelligent Transportation Systems. IEEE Trans. Intell. Transp. Syst. 2007, 8, 43-49. [CrossRef]

11. Raubal, M.; Winter, S. Enriching Wayfinding Instructions with Local Landmarks. In Boulder: International Conference on Geographic Information Science; Springer: Berlin, Heidelberg, 2002; pp. 243-259. 
12. Michon, P.E.; Denis, M. When and why are visual landmarks used in giving directions. In Spatial Information Theory; Volume 2205 of Lecture Notes in Computer, Science; Montello, D.R., Ed.; Springer: Berlin, Germany, 2001; pp. 292-305.

13. An, M.; Zhang, G.; Tao, D. Cognitive element analysis of spatial relationship of maps. J. Surv. Mapp. Sci. Technol. 2006, 23, 436-439.

14. Ungerer, F.; Schmid, H.J. An Introduction to Cognitive Linguistics; Foreign Teaching and Research Press: Beijing, China, 2001.

15. Roger, M.; Bonnardel, N.; Le Bigot, L. Landmarks' use in speech map navigation tasks. J. Environ. Psychol. 2011, 31, 192-199. [CrossRef]

16. Sweller, J. Instructional Design in Technical Areas; Australian Education Review: Camberwell, Australian, 1999.

17. Van Merrienboer, J.J.; Ayres, P. Research on Cognitive Load Theory and Its Design Implications for E-Learning. Educ. Technol. Res. Dev. 2005, 53, 5-13. [CrossRef]

18. Weng, M.; Jiang, S.; Qu, R. Mechanism of hierarchical spatial reasoning and its application in path finding. Sci. Surv. Mapp. 2006, 11, 119-121.

19. Zhao, W. Research on Intelligent Navigation Methods Based on Spatial Cognition. Ph.D. Thesis, Wuhan University, Wuhan, China, 2011; pp. 21-25.

20. Sun, C. Cognitive Load Measurement and Its Application in Multimedia Learning. 2012.

21. Donmez, B.; Cummings, M.L.; Graham, H.D.; Brzezinski, A.S. Modified Cooper Harper Scales for Assessing Unmanned Vehicle Displays. In Proceedings of the 10th Performance Metrics for Intelligent Systems Workshop, Baltimore, MD, USA, 28-30 September 2010; pp. 235-242.

22. Cooper, G.E.; Harper, P.J. The Use of Pilot Rating in the Evaluation of Air Craft Handing Qualities (Nasa Tn-D-5153); NASA: Washington, DC, USA, 1969.

23. Suzuki, K. Activities of the Japan society for graphic science-research and education. J. Geometry Graphics 2002, 6, 221-229.

24. Sarjakoski, T.; Kettunen, P.; Halkosaari, H.M.; Laakso, M.; Rönneberg, M.; Stigmar, H.; Sarjakoski, T. Landmarks and a hiking ontology to support wayfinding in a national park during different seasons. In Cognitive and Linguistic Aspects of Geographic Space; Springer: Berlin/Heidelberg, Germany, 2013; pp. 99-119.

25. Viaene, P.; Vanclooster, A.; Ooms, K.; De Maeyer, P. Thinking aloud in search of landmark characteristics in an indoor environment. In Proceedings of the 2014 Ubiquitous Positioning Indoor Navigation and Location Based Service (UPINLBS), Corpus Christ, TX, USA, 20-21 November 2014; Landmarks and a Hiking Ontology to Support Wayfinding in a National Park During Different Seasons. Geography Department, Ghent University: Ghent, Belgium; IEEE; pp. 103-110.

26. Lavie, N. Distracted and confused? Selective attention under load. Trends Cogn. Sci. 2005, 9, 75-82. [CrossRef]

27. Mayer, R.E. Nine ways to reduce cognitive load in multimedia learning. Educ. Psychol. 2003, 8, 43-52. [CrossRef]

28. Wang, J.; Zhu, Z. Research progress in cognitive psychology of visual attention selectivity. Appl. Psychol. 1997, 3, 58-64.

29. Yan, Y.; Long, Y.; Shen, Q.; Wang, J. Spatial cognitive analysis of electronic map considering gender difference. Geogr. Geogr. Inf. Sci. 2011, 27, 48-51.

(C) 2020 by the authors. Licensee MDPI, Basel, Switzerland. This article is an open access article distributed under the terms and conditions of the Creative Commons Attribution (CC BY) license (http://creativecommons.org/licenses/by/4.0/). 